\title{
PBW Basis of Quantized Universal Enveloping Algebras
}

\author{
By \\ Yoshihisa SAITO*
}

\section{$\S 0$. Introduction}

In [4], Lusztig constructed PBW bases of $U_{q}^{+}$. Then, he introduced the canonical base of $U_{q}^{+}[6]$ in case of A, D, E type. His results can be reformulated in case of $U_{q}^{-}$as follows.

Let $L$ be the sub $\boldsymbol{Z}[q]$-module of $U_{q}^{-}$generated by a PBW basis of $U_{q}^{-}$. This submodule is independent of the choice of the PBW basis. Let $\pi^{\prime}: L \rightarrow$ $L / q L$ be the canonical projection. Then the image $B$ of the PBW basis is a $\boldsymbol{Z}$-basis of $L / q L$ and it is independent of the choice of the PBW bases. Let - : $U_{q} \rightarrow U_{q}$ be the $\boldsymbol{Q}$-algebra involution defined by $e_{i} \mapsto e_{\imath}, f_{i} \mapsto f_{i}, q^{h} \mapsto q^{-h}$, $q \mapsto q^{-1}$. Then $\pi^{\prime}$ induces a $\boldsymbol{Z}$-module isomorphism $\pi^{\prime \prime}: L \cap \bar{L} \rightarrow L / q L, \boldsymbol{B}=$ $\left(\pi^{\prime \prime}\right)^{-1}(B)$ is a $\boldsymbol{Z}$-basis of $L \cap \bar{L}$ and $\boldsymbol{Z}[q]$-basis of $L$. Moreover each element of $\boldsymbol{B}$ is fixed by $-\boldsymbol{B}$ is called the canonical base of $U_{q}^{-}$.

On the other hand, in [1] Kashiwara constructed the global crystal base of $U_{q}^{-}$. Let $(L(\infty), B(\infty))$ be the crystal base of $U_{q}^{-}$and let $U_{\bar{Q}}^{-}$be the sub-Q $\left[q, q^{-1}\right]$ algebra of $U_{q}^{-}$generated by $f_{i}^{(n)}$. Then $U_{\bar{Q}} \cap L(\infty) \cap L(\infty)^{-} \rightarrow L(\infty) / q L(\infty)$ is an isomorphism. Let $G$ be the inverse of this isomorphism. Then $G(B(\infty))$ is a base of $U_{q}^{-}$and called the global crystal base of $U_{q}^{-}$.

In [7], Lusztig showed $\boldsymbol{B}=G(B(\infty))$ in the simply laced case.

In this paper, we show that the monomials of the root vectors form a base of $U_{q}^{-}$and they give a crystal base at $q=0$, when $g$ is an arbitrary finite dimensional semisimple Lie algebra.

In Section 1, we define the braid group action on the integrable $U_{q}$-module. Let $M$ be an integrable $U_{q}$-module. Then we shall define the automorphism $S_{\imath}$ of $M$ as follows :

$$
S_{i} v=\exp _{q_{i}^{-1}}\left(q_{i}^{-1} e_{i} t_{i}^{-1}\right) \exp _{q_{i}^{-1}}\left(-f_{i}\right) \exp _{q_{i}^{-1}}\left(q_{i} e_{i} t_{i}\right) q_{i}^{h_{i}\left(h_{i}+1\right) / 2} v \quad \text { for } \quad v \in M .
$$

Communicated by M. Kashiwara, April 1, 1993.

1991 Math. Subject Classification: 17B37, 81R50.

* Department of Mathematical Sciences, University of Tokyo, Hongo, Tokyo, 113, Japan. 
Here $\exp _{q}(x)$ denotes the $q$-analogue of the exponential function $\sum_{k=0}^{\infty}\left(q^{k(k-1) / 2} /[k] !\right) x^{k}$. The operator $q_{i}^{h_{i}\left(h_{i}+1\right) / 2}$ sends $u$ to $q_{i}^{m(m+1) / 2} u$ if $t_{i} u=q_{i}^{m} u$. Here $q_{i}=q^{\left(\alpha_{i}, a_{2}\right)}$ and $t_{i}=q^{\left(\alpha_{i}, \alpha_{2}\right) h_{i}}$. Since $q_{i}^{-1} e_{i} t_{i}^{-1}, f_{\imath}$ and $q_{i} e_{i} t_{i}$ act on $M$ in locally nilpotent way, $S_{i}$ is well-defined. Moreover $\exp _{q}(x) \exp _{q-1}(-x)=1$ implies that $S_{i}$ is invertible. There exists a unique automorphism $T_{\imath}$ of $U_{q}$ such that $S_{i}(x v)=\left(T_{\imath} x\right) S_{i} v$ for $x \in U_{q}$ and $v \in M$. This automorphism $T_{2}$ coincides with the automorphism $T_{i}$ introduced by Lusztig [4] with a small modification. In Proposition 1.4.1 we shall show that $\left\{S_{\imath}\right\}$ satisfies the braid relation. In Section 2, we show $\operatorname{Ker} e_{i}^{\prime}=T_{2} U_{q}^{-} \cap U_{q}^{-}$. ( $e_{i}^{\prime}$ is defined in 2.1.) This is the key of this paper. In Section 3, we shall give a relation of cystal base and the braid group action. Let $P$ be an element of $T_{i}^{-1} U_{q}^{-} \cap U_{q}^{-}$. We assume that $P$ belongs to $L(\infty)$ and $P \bmod q L(\infty)$ belongs to $B(\infty)$. Using the fact that Ker $e_{i}^{\prime}=$ $T_{\imath} U_{q}^{-} \cap U_{q}^{-}$, we show that $T_{\imath} P$ belongs to $L(\infty)$ and $T_{\imath} P \bmod q L(\infty)$ belongs to $B(\infty)$. Thus $f_{i}^{(k)} T_{\imath} P$ belongs again to $L(\infty)$ and gives a crystal base at $q=0$. In Section 4 , we introduce PBW basis $\left\{f^{k} ; k=\left(k_{1}, \cdots, k_{N}\right) \in \boldsymbol{Z}_{\geq 0}^{N}\right\}$. Chosing a reduced expression $s_{i_{1}} \cdots s_{i_{N}}$ of the longest element of the Weyl group we define

$$
f^{k}=f_{i_{1}}^{\left(k_{1}\right)} T_{i_{1}}\left(f_{i_{2}}^{\left(k_{2}\right)} T_{i_{2}}\left(\cdots f_{i_{N-1}}^{\left(k_{N-1}\right)} T_{i_{N-1}} f_{i_{N}}^{\left(k_{N}\right)}\right) \cdots\right) .
$$

By the consequence of Section 3 we show that $f^{k}$ forms a base of $L(\infty)$ and $\left\{f^{k} \bmod q L(\infty)\right\}=B(\infty)$ when $g$ is a finite-dimensional semisimple Lie algebra (Main Theorem). This generalizes the result of Lusztig [7].

This paper could not be written without Professor M. Kashiwara's guidance. The author would like to thank Professor M. Kashiwara.

\section{$\S 1$. Braid Group Action on Integrable Modules}

\subsection{The operator $\Phi$}

We follow the notations in $[1,2,3]$. For example, $g$ is a symmetrizable Kac-Moody Lie algebra, $\left\{\alpha_{i}\right\}_{\imath \in I}$ is the set of simple roots, $\boldsymbol{P}$ is a weight lattice, $U_{q}$ is the corresponding quantized universal enveloping algebra generated by $e_{i}, f_{i}, q^{h}\left(h \in \boldsymbol{P}^{*}\right)$, etc.

Let $U_{q}\left(s l_{2}\right)_{i}$ be the subalgebra of $U_{q}$ generated by $e_{i}, f_{i}, t_{i}=q^{\left(\alpha_{i}, \alpha_{i}\right) h_{i}}$.

Introduce the $\boldsymbol{Q}(q)$-algebra anti-automorphism * of $U_{q}$ by

$$
e_{i}^{*}=e_{i}, \quad f_{i}^{*}=f_{i}, \quad\left(q^{h}\right)^{*}=q^{-h} .
$$

We define the $\boldsymbol{Q}(q)$-algebra homomorphism $\Phi: U_{q} \rightarrow \operatorname{End}\left(U_{q}\right)$ by

$$
\Phi(x)(y)=\Sigma\left(S\left(x_{(2)}\right)\right) * y x_{(1)}^{*}
$$

where 
Then we have

$$
\Delta(x)=\sum x_{(1)} \otimes x_{(2)}
$$

$$
\begin{aligned}
& \Phi\left(e_{\imath}\right)(x)=t_{i}^{-1}\left[x, e_{\imath}\right] \\
& \Phi\left(f_{\imath}\right)(x)=x f_{\imath}-f_{\imath} t_{\imath} x t_{\imath}^{-1} \\
& \Phi\left(t_{\imath}\right)(x)=t_{\imath} x t_{\imath}^{-1}
\end{aligned}
$$

for $x \in U_{q}$

Lemma 1.1.1. For $i \neq j$

$$
\Phi\left(f_{i}^{(n)}\right)\left(f_{\jmath}\right)=\sum_{k=0}^{n}(-1)^{n-k} q_{i}^{(n-k)\left(-a_{\imath \jmath}-n+1\right)} f_{i}^{(n-k)} f_{\jmath} f_{i}^{(k)} .
$$

Proof. Let $A$ and $B$ be the endomorphisms of $U_{q}$ defined by $A x=x f_{\imath}$ and $B x=f_{2} t_{2} x t_{\imath}^{-1}$. Then we have

$$
\Phi\left(f_{\imath}\right)(x)=A x-B x
$$

for $x \in U_{q}$. The operators $A$ and $B$ satisfy the commutation relation:

$$
A B=q_{i}^{2} B A .
$$

By the $q$-analogue of the binomial formula, we obtain

$$
\begin{aligned}
\Phi\left(f_{\imath}^{n}\right) & =(A-B)^{n} \\
& =\sum_{k=0}^{n}(-1)^{n-k} q_{\imath}^{-k(n-k)}\left[\begin{array}{l}
n \\
k
\end{array}\right]_{\imath} A^{k} B^{n-k} . \\
\Phi\left(f_{\imath}^{(n)}\right)\left(f_{\jmath}\right) & =\sum_{k=0}^{n}(-1)^{n-k} q_{\imath}^{(-n+1)(n-k)} f_{\imath}^{(n-k)} t_{\imath}^{n-k} f_{\jmath} t_{\imath}^{-n+k} f_{\imath}^{(k)} \\
& =\sum_{k=0}^{n}(-1)^{n-k} q_{\imath}^{(n-k)\left(-a_{\imath}-n+1\right)} f_{i}^{(n-k)} f_{J} f_{\imath}^{(k)} .
\end{aligned}
$$

For $n=1-a_{\imath \jmath}$, the Serre relation implies

$$
\Phi\left(f_{i}^{\left(1-a_{i \jmath}\right)}\right)\left(f_{j}\right)=\sum_{k=0}^{-a_{\imath \jmath}+1}(-1)^{-a_{\imath \jmath}+1-k} f_{\imath}^{\left(-a_{\imath \jmath}+1-k\right)} f_{j} f_{\imath}^{(k)}=0 .
$$

Along with $\Phi\left(e_{\imath}\right) f_{\jmath}=0$, we conclude that $f_{\jmath}$ is $U_{q}\left(s l_{2}\right)_{2}$-finite and it is a highest weight vector. Here $U_{q}$ is regarded as a $U_{q}\left(s l_{2}\right)_{2}$-module through $\Phi$.

\subsection{Definition of $S_{\imath}$}

Let $V(l)\left(l \in \boldsymbol{Z}_{\geq 0}\right)$ be the irreducible $U_{q}\left(s l_{2}\right)_{2}$-module of dimension $l+1$. Let us take a highest weight vector $u_{0}^{(l)}$ of $V(l)$. Then we have

$$
\begin{aligned}
& e_{\imath} u_{0}^{(l)}=0, \\
& t_{\imath} u_{0}^{(l)}=q_{\imath}^{l} u_{0}^{(l)} .
\end{aligned}
$$


Let $u_{k}^{(l)}=f_{i}^{(k)} u_{0}^{(l)}$. Then we have

$$
V(l)=\bigoplus_{k=0}^{l} \boldsymbol{Q}(q) u_{k}^{(l)} .
$$

Next, we define the endomorphism $S_{\imath}$ of the vector space $V(l)$ by

$$
S_{\imath} v=\exp _{q_{i}^{-1}}\left(q_{\imath}^{-1} e_{\imath} t_{\imath}^{-1}\right) \exp _{q_{i}^{-1}}{ }^{-1}\left(-f_{i}\right) \exp _{q_{i}^{-1}}\left(q_{\imath} e_{\imath} t_{\imath}\right) q_{\imath}{ }^{{ }^{h}\left(h_{\imath}+1\right) / 2} v
$$

for $v \in V(l)$, where

$$
\exp _{q}(X)=\sum_{k=0}^{\infty} \frac{q^{k(k-1) / 2}}{[k] !} X^{k}
$$

The operator $q_{\imath}^{h_{\imath}\left(h_{\imath}+1\right) / 2}$ sends $u$ to $q_{i}^{m(m+1) / 2} u$ for a vector $u$ with $t_{i} u=q_{i}^{m} u$. Since the action of $e_{\imath}$ and $f_{i}$ are nilpotent, this endomorphism is well defined.

For $x \in U_{q}$ and $n \geqq 0$, we set

$$
\begin{aligned}
x_{\imath} & =\frac{x-x^{-1}}{q_{\imath}-q_{\imath}^{-1}} \\
\left\{\begin{array}{l}
x \\
n
\end{array}\right\}_{i} & =\frac{\Pi_{k=1}^{n}\left(q_{i}^{1-k} x\right)_{2}}{[n]_{2} !} .
\end{aligned}
$$

Hence we have

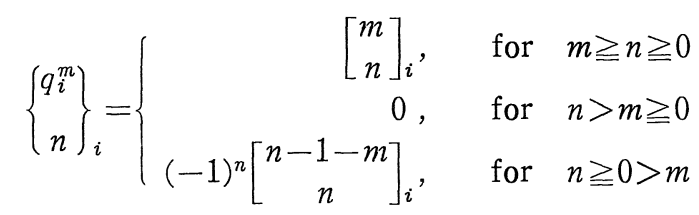

and

$$
\left\{\begin{array}{l}
x \\
n
\end{array}\right\}_{\imath}=(-1)^{n}\left\{\begin{array}{c}
q_{i}^{n-1} x^{-1} \\
n
\end{array}\right\}_{\imath} .
$$

Ordinary, $\left[\begin{array}{l}m \\ n\end{array}\right]_{i}$ is defined for $m \geqq 0, n \geqq 0$. But we will extend $\left[\begin{array}{l}m \\ n\end{array}\right]_{i}$ for $m \in Z$, $n \geqq 0$. We set

$$
\left[\begin{array}{l}
m \\
n
\end{array}\right]_{i}=\left\{\begin{array}{l}
q_{i}^{m} \\
n
\end{array}\right\}_{\imath}
$$

Then we have

$$
\left[\begin{array}{l}
m \\
n
\end{array}\right]_{i}=(-1)^{n}\left[\begin{array}{c}
n-1-m \\
n
\end{array}\right]_{i}
$$

Proposition 1.2.1.

$$
S_{i} u_{k}^{(l)}=(-1)^{l-k} q_{i}^{(l-k)(k+1)} u_{l-k}^{(l)}
$$

for any $0 \leqq k \leqq l$.

The first step is the next lemma. 
Lemma 1.2.2. For integers $s, k, l$ with $0 \leqq k, s \leqq l$, we have

$$
\begin{gathered}
\sum(-1)^{b} q^{(l-2 k)(c-a)-c(b-c)+b-a(a-b+c)}\left[\begin{array}{c}
l-k+c \\
c
\end{array}\right]\left[\begin{array}{c}
l-s+a \\
b
\end{array}\right]\left[\begin{array}{l}
s \\
a
\end{array}\right] \\
=(-1)^{l-k} q^{(l-k)(k+1)} \delta_{k, s}
\end{gathered}
$$

where the sum ranges over non-negative integers $a, b, c$ such that $s=l-k+a-b+c$.

Proof. We recall the next formula

$$
\sum_{n=0}^{k} x^{-n} y^{k-n}\left\{\begin{array}{l}
y \\
n
\end{array}\right\}\left\{\begin{array}{c}
x \\
k-n
\end{array}\right\}=\left\{\begin{array}{c}
x y \\
k
\end{array}\right\}
$$

for $x, y \in U_{q}$. Then, (1.2.5) and (1.2.8) imply

$$
\sum_{n=0}^{k} q^{-a n+b(k-n)}\left[\begin{array}{l}
b \\
n
\end{array}\right]\left[\begin{array}{c}
a \\
k-n
\end{array}\right]=\left[\begin{array}{c}
a+b \\
k
\end{array}\right] .
$$

By (1.2.6) and (1.2.9), we have

$$
\begin{aligned}
\Sigma(-1)^{c} q^{-c(l-s+a)+(k-c)(-l+k-1)}\left[\begin{array}{c}
l-k+c \\
c
\end{array}\right]\left[\begin{array}{c}
l-s+a \\
k-c
\end{array}\right] \\
=\sum q^{-c(l-s+a)+(k-c)(-l+k-1)}\left[\begin{array}{c}
-l+k-1 \\
c
\end{array}\right]\left[\begin{array}{c}
l-s+a \\
k-c
\end{array}\right] \\
=\left[\begin{array}{c}
k+a-s-1 \\
k
\end{array}\right] \\
=(-1)^{k}\left[\begin{array}{c}
s-a \\
k
\end{array}\right] \\
=(-1)^{k}\left[\begin{array}{c}
s-a \\
s-a-k
\end{array}\right] \\
=(-1)^{s-a}\left[\begin{array}{l}
-k-1 \\
s-a-k
\end{array}\right]
\end{aligned}
$$

and

Therefore

$$
\begin{aligned}
\Sigma q^{s(s-a-k)+a(k+1)}\left[\begin{array}{c}
-k-1 \\
s-a-k
\end{array}\right]\left[\begin{array}{l}
s \\
a
\end{array}\right] & =\left[\begin{array}{c}
s-k-1 \\
s-k
\end{array}\right] \\
& =\delta_{s, k} .
\end{aligned}
$$

$$
\Sigma(-1)^{c+s-a} q^{-c(l-s+a)+(k-c)(-l+k-1)+s(s-a-k)+a(k+1)}\left[\begin{array}{c}
l-k+c \\
c
\end{array}\right]\left[\begin{array}{c}
l-s+a \\
b
\end{array}\right]\left[\begin{array}{l}
s \\
a
\end{array}\right]=\delta_{s, k} .
$$


Assume that $s$ equals $k$. Since $s=l-k+a-b+c$, we have

$$
\begin{aligned}
& -c(l-s+a)+(k-c)(-l+k-1)+s(s-a-k)+a(k+1) \\
& \quad=(l-2 k)(c-a)-c(b-c)+b-a(a-b+c)-(k+1)(l-k),
\end{aligned}
$$

which implies

$$
\sum(-1)^{b} q^{(l-2 k)(c-a)-c(b-c)+b-a(a-b+c)}\left[\begin{array}{c}
l-k+c \\
c
\end{array}\right]\left[\begin{array}{c}
l-s+a \\
b
\end{array}\right]\left[\begin{array}{l}
s \\
a
\end{array}\right]=(-1)^{l-k} q^{(l-k)(k+1)} \delta_{s, k} .
$$

We proved the lemma.

Q.E.D.

We define the endomorphism $s_{\imath}$ of $V(l)$ by

$$
s_{\imath}=\sum_{a, b, c}(-1)^{b} q_{i}^{c(c-b)-a(a-b+c)+b} e_{i}^{(a)} f_{i}^{(b)} e_{i}^{(c)} t_{i}^{c-a} .
$$

\section{Lemma 1.2.3.}

$$
s_{i} u_{k}^{(l)}=(-1)^{l-k} q_{i}^{(l-k)(k+1)} u_{l-k}^{(l)} .
$$

Proof. We get

$$
s_{i} u_{k}^{(l)}=\Sigma(-1)^{b} q_{i}^{(l-2 k)(c-a)+c(c-b)+b-a(a-b+c)}\left[\begin{array}{c}
l-k+c \\
c
\end{array}\right]_{i}\left[\begin{array}{c}
l-s+a \\
b
\end{array}\right]_{i}\left[\begin{array}{l}
s \\
a
\end{array}\right]_{i} u_{l-s}^{(l)} .
$$

Indeed

$$
\begin{aligned}
s_{\imath} u_{k}^{(l)}= & \Sigma(-1)^{b} q_{i}^{c(c-b)-a(a-b+c)+b} e_{i}^{(a)} f_{i}^{(b)} e_{i}^{(c)}\left(q_{i}^{(l-2 k)(c-a)}\right) u_{k}^{(\imath)} \\
& =\Sigma(-1)^{b} q_{i}^{(l-2 k)(c-a)+c(c-b)+b-a(a-b+c)} \\
& {\left[\begin{array}{c}
l-k+c \\
c
\end{array}\right]_{\imath}\left[\begin{array}{c}
k-c+b \\
b
\end{array}\right]_{i}\left[\begin{array}{c}
l-k+c-b+a \\
a
\end{array}\right]_{\imath} u_{k-a+b-c}^{(l)} } \\
& =\Sigma(-1)^{b} q_{i}^{(l-2 k)(c-a)+c(c-b)+b-a(a-b+c)}\left[\begin{array}{c}
l-k+c \\
c
\end{array}\right]_{i}\left[\begin{array}{c}
l-s+a \\
b
\end{array}\right]_{i}\left[\begin{array}{l}
s \\
a
\end{array}\right]_{i} u_{l-s}^{(l)}
\end{aligned}
$$

where $s=l-k+c-b+a$. By Lemma 1.2.2, we have

$$
s_{i} u_{k}^{(l)}=(-1)^{l-k} q_{i}^{(l-k)(k+1)} u_{l-k}^{(l)} .
$$

Q.E.D.

Proof of Proposition 1.2.1. It is enough to show that $s_{i}$ equals $S_{i}$. First, we get

$$
e_{i}^{(a)} f_{i}^{(b)} e_{i}^{(c)} t_{i}^{c-a}=q_{i}^{2 a c-2 a b+a(a-1)-c(c-1)} \frac{\left(e_{i} t_{i}^{-1}\right)^{a}}{[a]_{i} !} f_{i}^{(b)} \frac{\left(e_{i} t_{i}\right)^{c}}{[c]_{i} !} .
$$

Then, we have 


$$
s_{\imath}=\sum(-1)^{b} q_{\imath}^{c(c-b)-a(a-b+c)+b+2 a c-2 a b+a(a-1)-c(c-1)} \frac{\left(e_{2} t_{\imath}^{-1}\right)^{a}}{[a]_{\imath} !} f_{i}^{(b)} \frac{\left(e_{2} t_{\imath}\right)^{c}}{[c]_{i} !} .
$$

On the other hand, we have

$$
\begin{aligned}
& c(c-b)-a(a-b+c)+b+2 a c-2 a b+a(a-1)-c(c-1) \\
& \quad=-\frac{a(a-1)}{2}-a-\frac{b(b-1)}{2}-\frac{c(c-1)}{2}+c+\frac{(-a+b-c)(-a+b-c+1)}{2} .
\end{aligned}
$$

Therefore, we have

$$
\begin{aligned}
& s_{\imath} u_{k}^{(l)}=\sum_{a, b, c} \frac{q_{i}^{-a}(a-1) / 2}{[a]_{\imath} !}\left(q_{\imath}^{-1} e_{\imath} t_{\imath}^{-1}\right)^{a} \frac{q_{l}^{-b(b-1) / 2}}{[b]_{\imath} !} f_{\imath}^{b} \frac{q_{l}^{-c(c-1) / 2}}{[c]_{i} !}\left(q_{\imath} e_{\imath} t_{\imath}\right)^{c} q_{i}^{(-a+b-c)(-a+b-c+1) / 2} u_{k}^{(l)} \\
& =S_{i} u_{k}^{(l)} \text {, }
\end{aligned}
$$

We define the endomorphism $S_{\imath}^{\prime}$ of $V(l)$ by

$$
S_{\imath}^{\prime} u_{k}^{(l)}=\exp _{q_{i}^{-1}}\left(-q_{\imath}^{-1} f_{\imath} t_{\imath}\right) \exp _{q_{\imath}^{-1}}\left(e_{\imath}\right) \exp _{q_{\imath}^{-1}}\left(-q_{\imath} f_{\imath} t_{\imath}^{-1}\right) q_{\imath}^{h_{\imath}\left(h_{\imath}+1\right) / 2} .
$$

We can prove the next result similarly,

$$
S_{i}^{\prime} u_{k}^{(l)}=(-1)^{l-k} q_{\imath}^{(l-k)(k+1)} u_{l-k}^{(l)} .
$$

Therefore, $S_{\imath}=S_{\imath}^{\prime}$, and (1.2.13) is another expression of $S_{\imath}$.

Let $M$ be an integrable $U_{q}$-module. $M$ is a direct sum of irreducible $U_{q}\left(s l_{2}\right)_{2}$ modules. So, we regard $S_{\imath}$ as an endomorphism of $M$.

\subsection{Definition of $T_{2}$}

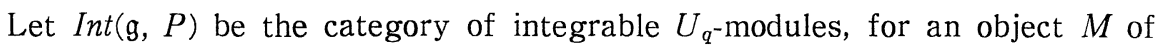
Int $(\mathrm{g}, P)$ let $\Psi(M)$ be the underlying $\boldsymbol{Q}(q)$-vectorspace. Then $\Psi$ is the functor from $\operatorname{Int}(\mathrm{g}, P)$ to the category of $\boldsymbol{Q}(q)$ vector spaces. Let $R$ be the endomorphism ring. Then $R$ contains $S_{\imath}$ as well as $U_{q}$.

We define the algebra automorphism $T_{\imath}$ of $R$ by

$$
T_{\imath} x=\operatorname{Ad} S_{\imath}(x)
$$

for $x \in U_{q}$.

Proposition 1.3.1. We have

$$
\begin{aligned}
& T_{\imath}\left(e_{\imath}\right)=-f_{\imath} t_{\imath} \\
& T_{i}\left(f_{\imath}\right)=-t_{i}^{-1} e_{\imath} \\
& T_{\imath}\left(t_{\jmath}\right)=t_{\jmath} t_{i}^{-a_{i \jmath}}
\end{aligned}
$$




$$
\begin{aligned}
& T_{\imath}\left(e_{\jmath}\right)=\sum_{k=0}^{-a_{i \jmath}}(-1)^{-a_{\imath j^{-k}}} q_{\imath}^{a_{\imath j^{+k}}} e_{i}^{(k)} e_{\jmath} e_{i}^{\left(-a_{\imath \jmath^{-k}}\right)} \quad \text { for } \quad i \neq j \\
& T_{\imath}\left(f_{\jmath}\right)=\sum_{k=0}^{-a_{\imath \jmath}}(-1)^{-a_{i \jmath^{-k}}} q_{i}^{-a_{\imath j^{-k}}} f_{i}^{\left(-a_{\imath \jmath^{-k}}\right)} f_{\jmath} f_{i}^{(k)} \quad \text { for } \quad i \neq j .
\end{aligned}
$$

In particular $U_{q}$ is stable by $T_{\imath}$.

Proof. Let $\left\{u_{k}^{(l)}\right\}$ be as in 1.2. Then we have

$$
\begin{aligned}
S_{\imath} e_{\imath} u_{k}^{(l)} & =S_{\imath} e_{i} S_{\imath}^{-1} S_{\imath} u_{k}^{(l)} \\
& =T_{\imath}\left(e_{\imath}\right)(-1)^{l-k} q_{i}^{(l-k)(k+1)} u_{l-k}^{(l)}
\end{aligned}
$$

and

$$
\begin{aligned}
S_{\imath} e_{\imath} u_{k}^{(l)} & =[l-k+1]_{\imath} S_{\imath} u_{k-1}^{(l)} \\
& =[l-k+1]_{\imath}(-1)^{l-k+1} q_{\imath}^{k(l-k+1)} u_{l-k+1}^{(l)} .
\end{aligned}
$$

Therefore $T_{\imath}\left(e_{\imath}\right)=-f_{\imath} t_{\imath}$. Similarly, $T_{\imath}\left(f_{\imath}\right)=-t_{\imath}^{-1} e_{\imath}$ and $T_{\imath}\left(t_{j}\right)=t_{j} t_{\imath}^{-a_{\imath j}}$. We shall prove (1.3.6).

Lemma 1.3.2. Let

$$
S_{i}^{\prime \prime}=\exp _{q_{i}^{-1}}\left(-f_{\imath}\right) \exp _{q_{\imath}^{-1}}\left(q_{\imath} e_{\imath} t_{\imath}\right) .
$$

Then we have

$$
\operatorname{Ad}\left(S_{\imath}^{\prime \prime}\right)\left(f_{\jmath} t_{j}^{-1}\right)=q_{\imath}^{-a_{\imath \jmath}\left(a_{\imath \jmath}-1\right) / 2} \operatorname{Ad}\left(\exp _{q_{i}}\left(-q_{i}^{-1} e_{\imath} t_{i}^{-1}\right)\right)\left(\Phi\left(f_{i}^{\left(-a_{\imath j}\right)}\right)\left(f_{\jmath}\right) t_{j}^{-1}\right) .
$$

Proof. Since $\left[q_{2} e_{2} t_{2}, f_{j} t_{j}^{-1}\right]=0$, we get

$$
\operatorname{Ad}\left(\exp _{q_{i}^{-1}}\left(q_{\imath} e_{\imath} t_{\imath}\right)\right)\left(f_{j} t_{j}^{-1}\right)=f_{j} t_{j}^{-1} .
$$

Therefore

$$
\begin{aligned}
\operatorname{Ad}\left(S_{\imath}^{\prime \prime}\right)\left(f_{\jmath} t_{j}^{-1}\right) & =\operatorname{Ad}\left(\exp _{q_{\imath}}-1\left(-f_{\imath}\right)\right)\left(f_{j} t_{j}^{-1}\right) \\
& =\sum_{n=0}^{\infty} \sum_{k=0}^{n}(-1)^{n-k} q_{\imath}^{-(n(n-1) / 2)+k\left(n-1+a_{\imath j}\right)} f_{i}^{(n-k)} f_{\jmath} f_{i}^{(k)} t_{j}^{-1} \\
& =\sum_{n=0}^{\infty} q_{\imath}^{-(n(n-1) / 2)-n\left(-a_{\imath},-n+1\right)} \Phi\left(f_{i}^{(n)}\right)\left(f_{\jmath}\right) t_{j}^{-1} \\
& =\sum_{n=0}^{-a i_{j}} q_{i}^{(n(n-1) / 2)+n a_{i j}} \Phi\left(f_{i}^{(n)}\right)\left(f_{j}\right) t_{j}^{-1} .
\end{aligned}
$$

By (1.1.3) and Lemma 1.1.1, $\bigoplus_{k=0}^{-a} i_{j} \boldsymbol{Q}(q) \Phi\left(f_{i}^{(k)}\right)\left(f_{\jmath}\right)$ is a $\left(-a_{\imath \jmath}+1\right)$-dimensional irreducible $U_{q}\left(s l_{2}\right)_{\imath}$-submodule of $U_{q}$, and $f_{j}$ is a highest weight vector of weight $-a_{\imath \jmath}$. Therefore

$$
\Phi\left(e_{i}^{(k)}\right) \Phi\left(f_{i}^{\left(-a_{\imath j}\right)}\right)\left(f_{\jmath}\right)=\Phi\left(f_{i}^{\left(-a_{i j}-k\right)}\right)\left(f_{\jmath}\right)
$$

Then, we have 


$$
\begin{aligned}
& \operatorname{Ad}\left(\exp _{q_{i}}\left(-q_{i}^{-1} e_{\imath} t_{i}^{-1}\right)\right)\left(\Phi\left(f_{i}^{\left(-a_{\imath j}\right)}\right)\left(f_{\jmath}\right) t_{j}^{-1}\right) \\
& \quad=\sum_{n=0}^{\infty} \sum_{k=0}^{n}(-1)^{k} q_{i}^{(n(n+1) / 2)+k(n-1)} t_{i}^{-n} e_{i}^{(k)}\left(\Phi\left(f_{i}^{\left(-a_{\imath \jmath}\right)}\right)\left(f_{\jmath}\right)\right) e_{i}^{(n-k)} t_{j}^{-1} \\
& =\sum_{n=0}^{\infty} q_{\imath}^{(n+1) / 2} \Phi\left(e_{i}^{(n)}\right) \Phi\left(f_{i}^{\left(-a_{i \jmath}\right)}\right)\left(f_{j}\right) t_{j}^{-1} \\
& =\sum_{n=0}^{-a_{\imath \jmath}} q_{\imath}^{n(n+1) / 2} \Phi\left(f_{i}^{\left(-a_{\imath \jmath^{-n}}\right)}\right)\left(f_{j}\right) t_{j}^{-1} \\
& =\sum_{n=0}^{-a_{\imath \jmath}} q_{i}^{(n(n-1) / 2)+n a_{\imath j^{+}}\left(a_{i j}\left(a_{\imath j^{-1) / 2}}\right)\right.} \Phi\left(f_{i}^{(n)}\right)\left(f_{\jmath}\right) t_{j}^{-1} .
\end{aligned}
$$

Q.E.D.

Let

$$
S_{\imath}^{\prime}=\exp _{q_{\imath}^{-1}}\left(q_{\imath}^{-1} e_{\imath} t_{i}^{-1}\right) S_{\imath}^{\prime \prime} .
$$

By Lemma 1.3.2, we have

$$
\operatorname{Ad}\left(S_{i}^{\prime}\right)\left(f_{\jmath} t_{j}^{-1}\right)=q_{\imath}^{-a_{\imath j}\left(a_{\imath \jmath}-1\right) / 2} \Phi\left(f_{\imath}^{\left(-a_{\imath \jmath}\right)}\right)\left(f_{\jmath}\right) t_{j}^{-1} .
$$

On the other hand, we get

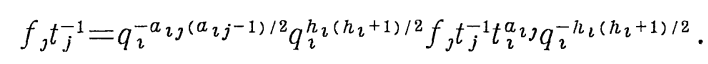

Therefore,

$$
\begin{aligned}
\operatorname{Ad}\left(S_{i}^{\prime}\right)\left(f_{\jmath} t_{j}^{-1}\right) & =q_{\imath}^{-a_{i j}\left(a_{\imath \jmath}-1\right) / 2} \operatorname{Ad}\left(S_{\imath}\right)\left(f_{\jmath} t_{j}^{-1} t_{\imath}^{a_{\imath \jmath}}\right) \\
& =q_{i}^{-a_{\imath j\left(a_{\imath \jmath}-1\right) / 2}} \operatorname{Ad}\left(S_{\imath}\right)\left(f_{\jmath}\right) t_{j}^{-1} .
\end{aligned}
$$

This and (1.3.9) imply (1.3.6).

Introduce the $\boldsymbol{Q}$-algebra anti-automorphism $\boldsymbol{\omega}$ by

$$
\begin{aligned}
\omega e_{\imath} & =f_{\imath} \\
\omega f_{\imath} & =e_{\imath} \\
\omega t_{\imath} & =t_{\imath}^{-1} \\
\omega q & =q^{-1} .
\end{aligned}
$$

Applying $\omega$ to (1.3.6), we obtain (1.3.5).

Q.E.D.

This proposition immediately imply the next corollary.

Corollary 1.3.3.

$$
* T_{\imath} *=T_{\imath}^{-1} \text {. }
$$

\subsection{The braid group action}

Proposition 1.4.1. $\left\{S_{\imath} ; i \in I\right\}$ satisfies the braid relations for the Weyl group 
$W$ of $\mathfrak{g}$.

Proof. In case of $a_{\imath \jmath}=a_{\jmath 2}=-1$, we have to prove

$$
S_{\imath} S_{\jmath} S_{\imath}=S_{\jmath} S_{i} S_{\jmath} .
$$

This is equivalent to

$$
S_{\jmath}=\left(\operatorname{Ad} S_{\imath}\right)\left(\operatorname{Ad} S_{\jmath}\right)\left(S_{i}\right)=T_{\imath} T_{\jmath}\left(S_{\imath}\right) .
$$

By (1.3.2)-(1.3.6), we have

$$
\begin{aligned}
& T_{\imath} T_{\jmath}\left(f_{\imath}\right)=f_{\jmath} \\
& T_{\imath} T_{\jmath}\left(e_{i}\right)=e_{\jmath} \\
& T_{\imath} T_{\jmath}\left(t_{\imath}\right)=t_{\jmath} .
\end{aligned}
$$

Therefore we get

$$
\begin{aligned}
& T_{\imath} T_{\jmath}\left(\exp _{q_{\imath}^{-1}}\left(q_{\imath}^{-1} e_{\imath} t_{\imath}^{-1}\right) \exp _{q_{i}^{-1}}\left(-f_{\imath}\right) \exp _{\left.q_{\imath}^{-1}\left(q_{\imath} e_{\imath} t_{\imath}\right) q_{i}^{h} i^{\left(h_{\imath}+1\right) / 2}\right)}\right. \\
& =\exp _{q_{i}^{-1}}{ }^{-1}\left(q_{\imath}^{-1} T_{\imath} T_{\jmath}\left(e_{\imath} t_{\imath}^{-1}\right)\right) \exp _{q_{\imath}^{-1}}\left(-T_{\imath} T_{\jmath}\left(f_{\imath}\right)\right) \exp _{q_{\imath}^{-1}}\left(q_{\imath} T_{\imath} T_{\jmath}\left(e_{\imath} t_{\imath}\right)\right) q_{\imath}^{h_{\jmath}\left(h_{\jmath}+1\right) / 2} \\
& =\exp _{q_{j}^{-1}}\left(q_{j}^{-1} e_{\jmath} t_{j}^{-1}\right) \exp _{q_{j}^{-1}}\left(-f_{\jmath}\right) \exp _{q_{j}^{-1}}\left(q_{\jmath} e_{j} t_{\jmath}\right) q_{j}^{h_{\jmath}\left(h_{\jmath}+1\right) / 2} \\
& =S_{\jmath} \text {. }
\end{aligned}
$$

The remaining cases are similarly proved by the corresponding identities to (1.4.1) due to Lusztig [4].

Q.E.D.

This proposition immediately implies the following result.

Corollary 1.4.2 ([5]). $\left\{T_{\imath} ; i \in I\right\}$ satisfies the braid relations.

\section{§2. $T_{\imath} U_{q}^{-} \cap U_{q}^{-}=\operatorname{Ker} e_{\imath}^{\prime}$}

\subsection{Proof of $T_{2} U_{q}^{-} \cap U_{q}^{-}=\operatorname{Ker} e_{\imath}^{\prime}$}

Let $U_{q}^{-}$be the subalgebla over $\boldsymbol{Q}(q)$ of $U_{q}$ generated by $f_{\imath}$.

Lemma 2.1.1 ([1]. For any $P \in U_{q}^{-}$, there exist unique $Q, R \in U_{q}^{-}$such that

$$
\left[e_{\imath}, P\right]=\frac{t_{\imath} Q-t_{\imath}^{-1} R}{q_{\imath}-q_{i}^{-1}} .
$$

By this lemma, if we set $e_{i}^{\prime \prime}(P)=Q$ and $e_{i}^{\prime}(P)=R$, then $e_{i}^{\prime}$ and $e_{i}^{\prime \prime}$ are endomorphism of $U_{\bar{q}}^{-}$. Moreover, we get

and

$$
e_{i}^{\prime \prime} f_{\jmath}=q_{i}^{a} i_{j} f_{\jmath} e_{i}^{\prime \prime}+\delta_{\imath \jmath}
$$

$$
e_{i}^{\prime} f_{\jmath}=q_{i}^{-a_{i j}} f_{\jmath} e_{i}^{\prime}+\delta_{i \jmath} .
$$

Here $f$, acts on $U_{q}^{-}$by the left multiplication. 
The aim of this section is to prove the following result.

\section{Proposition 2.1.2.}

$$
T_{\imath}\left(U_{q}^{-}\right) \cap U_{q}^{-}=\operatorname{Ker} e_{i}^{\prime} .
$$

The first step is the next lemma.

\section{Lemma 2.1.3.}

$$
f_{\imath}\left(U_{q}^{-}\right) \cap T_{\imath}\left(U_{q}^{-}\right)=\{0\}
$$

Proof. Let $u \in f_{i}\left(U_{q}^{-}\right) \cap T_{\imath}\left(U_{q}^{-}\right)$. Then $T_{i}^{-1} u \in U_{q}^{-}$. On the other hand, choosing $x \in U_{q}^{-}$such that $u=f_{2} x$, we have

$$
T_{\imath}^{-1} u=-e_{\imath} t_{\imath} T_{\imath}^{-1} x \text {. }
$$

Since $e_{\imath} U_{q} \cap U_{q}^{-}=0$, we obtain $u=0$.

Q.E.D.

Lemma 2.1.4. For $i-\leqslant j$

$$
f_{\jmath} f_{i}^{(n)}=\sum_{k=0}^{n} q_{i}^{k\left(n-k-a_{i j}\right)} f_{i}^{(k)} \Phi\left(f_{i}^{(n-k)}\right)\left(f_{j}\right) .
$$

Proof. By the definition of $\Phi$ we have

$$
\Phi\left(f_{\imath}\right)\left(\Phi\left(f_{i}^{(n)}\right)\left(f_{\jmath}\right)\right)=\Phi\left(f_{i}^{(n)}\right)\left(f_{\jmath}\right) f_{2}-f_{2} t_{2} \Phi\left(f_{i}^{(n)}\right)\left(f_{\jmath}\right) t_{\imath}^{-1} .
$$

Therefore we have

$$
\Phi\left(f_{i}^{(n)}\right)\left(f_{\jmath}\right) f_{i}=[n+1]_{\imath} \Phi\left(f_{i}^{(n+1)}\right)\left(f_{j}\right)+q_{\imath}^{2 n-a_{i j}} f_{\imath} \Phi\left(f_{i}^{(n)}\right)\left(f_{\jmath}\right) .
$$

We shall show this formula by induction on $n$,

$$
\begin{aligned}
f_{\jmath} f_{i}^{(n+1)}= & \frac{1}{[n+1]_{i}}\left(\sum_{k=0}^{n} q_{i}^{k\left(n-k-a_{i j}\right)} f_{i}^{(k)} \Phi\left(f_{i}^{(n-k)}\right)\left(f_{\jmath}\right)\right) f_{\imath} \\
= & \frac{1}{[n+1]_{i}} \sum_{k=0}^{n} q_{i}^{k\left(n-k-a_{i j}\right)} f_{i}^{(k)}\left([n-k+1]_{i} \Phi\left(f_{i}^{(n-k+1)}\right)\left(f_{\jmath}\right)\right. \\
& \left.+q_{i}^{2(n-k)-a_{i j}} f_{i} \Phi\left(f_{i}^{(n-k)}\right)\left(f_{\jmath}\right)\right) \\
= & \frac{1}{[n+1]_{i}} \sum_{k=0}^{n} q_{i}^{k\left(n-k-a_{i j}\right)}[n-k+1]_{i} f_{i}^{(k)} \Phi\left(f_{i}^{(n-k+1)}\right)\left(f_{\jmath}\right) \\
& +\frac{1}{[n+1]_{i}} \sum_{k=1}^{n+1} q_{i}^{k\left(n-k-a_{i j}\right)+n+1}[k]_{i} f_{i}^{(k)} \Phi\left(f_{i}^{(n-k+1)}\right)\left(f_{\jmath}\right) \\
= & \sum_{k=0}^{n+1} q_{i}^{k\left(n+1-k-a_{i j)}\right)} f_{i}^{(k)} \Phi\left(f_{i}^{(n-k+1)}\right)\left(f_{\jmath}\right) .
\end{aligned}
$$

\section{Corollary 2.1.5.}

$$
U_{q}^{-}=f_{i}\left(U_{q}^{-}\right)+M
$$


where $M$ is the $\boldsymbol{Q}(q)$-subalgebra of $U_{q}^{-}$generated by $\Phi\left(f_{i}^{(n)}\right)\left(f_{j}\right)$ for $i \neq j$ and $n \geqq 0$.

Lemma 2.1.6. For $i \neq j$

$$
T_{i}^{-1} \Phi\left(f_{i}^{(n)}\right)\left(f_{\jmath}\right)=\left(\Phi\left(f_{i}^{\left(-a_{i j^{-n}}\right)}\right)\left(f_{j}\right)\right)^{*} .
$$

Proof. We shall show this formula by induction on $n$,

$$
\begin{aligned}
& T_{i}^{-1} \Phi\left(f_{i}^{(n+1)}\right)\left(f_{\jmath}\right) \\
& =\frac{1}{[n+1]_{\imath}} T_{\imath}\left(\Phi\left(f_{\imath}\right) \Phi\left(f_{i}^{(n)}\right)\left(f_{\jmath}\right)\right) \\
& =\frac{1}{[n+1]_{\imath}} T_{\imath}\left(\Phi\left(f_{i}^{(n)}\right)\left(f_{\jmath}\right) f_{\imath}-q_{\imath}^{-2 n-a_{i j} f_{i}} \Phi\left(f_{i}^{(n)}\right)\left(f_{j}\right)\right)
\end{aligned}
$$

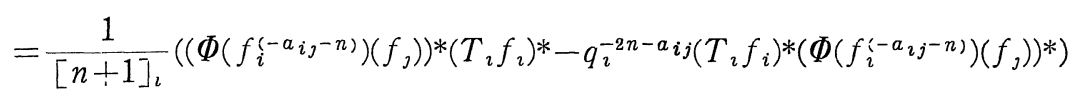

$$
\begin{aligned}
& =\frac{1}{[n+1]_{\imath}}\left(-t_{\imath}^{-1} e_{\imath} \Phi\left(f_{i}^{\left(-a_{\imath j}-n\right)}\right)\left(f_{\jmath}\right)+q_{\imath}^{-2 n-a_{i j}} \Phi\left(f_{i}^{\left(-a_{\imath j}-n\right)}\right)\left(f_{\jmath}\right) t_{\imath}^{-1} e_{i}\right)^{*} \\
& =\frac{1}{[n+1]_{\imath}}\left(\Phi\left(e_{2}\right) \Phi\left(f_{i}^{\left(-a_{i j}-n\right)}\right)\left(f_{\jmath}\right)\right)^{*} \\
& =\left(\Phi\left(f_{i}^{\left(-a_{\imath j}-n-1\right)}\right)\left(f_{\jmath}\right)\right) * \text {. }
\end{aligned}
$$

Corollary 2.1.7. For $i \neq j$,

$$
\Phi\left(f_{i}^{(n)}\right)\left(f_{j}\right) \in T_{i}\left(U_{q}^{-}\right) \cap U_{q}^{-} .
$$

Proof. $U_{q}^{-}$is stable under $*$. Lemma 2.1 .6 immediately implies this corollary.

Q. E. D.

Lemma 2.1.8. For $i \pm j$

$$
e_{i}^{\prime} \Phi\left(f_{i}^{(n)}\right)\left(f_{j}\right)=q_{i}^{-2 n-a_{i j}} \Phi\left(f_{i}^{(n)}\right)\left(f_{j}\right) e_{i}^{\prime} .
$$

Proof. First we have

Then, we have

$$
e_{i}^{\prime} f_{i}^{(n)}=q_{i}^{-2 n} f_{i}^{(n)} e_{i}^{\prime}+q_{i}^{1-n} f_{i}^{(n-1)} .
$$

$$
\begin{aligned}
e_{i}^{\prime} f_{i}^{(n-k)} f_{j} f_{i}^{(k)}= & q_{i}^{-2 n-a_{i j}} f_{i}^{(n-k)} f_{j} f_{i}^{(k)} e_{i}^{\prime}+q_{\imath}^{-2 n+k+1-a_{i j}} f_{i}^{(n-k)} f_{j} f_{i}^{(k-1)} \\
& +q_{i}^{-n+k+1} f_{i}^{(n-k-1)} f_{j} f_{i}^{(k)} .
\end{aligned}
$$

Therefore

$$
\begin{aligned}
e_{i}^{\prime} \Phi\left(f_{i}^{(n)}\right)\left(f_{\jmath}\right) & =\sum_{k=0}^{n}(-1)^{n-k} q_{i}^{(n-k)\left(-a_{i j^{-n+1}}\right.} e_{i}^{\prime} f_{i}^{(n-k)} f_{j} f_{i}^{(k)} \\
& =q_{i}^{-2 n-a_{i j}} \Phi\left(f_{i}^{(n)}\right)\left(f_{\jmath}\right) e_{i}^{\prime}
\end{aligned}
$$




$$
\begin{aligned}
& +\sum_{k=0}^{n}(-1)^{n-k} q_{i}^{(n-k)\left(-a_{\imath \jmath}-n+1\right)} \\
& \cdot\left(q_{i}^{-2 n+k+1-a_{i j}} f_{i}^{(n-k)} f_{\jmath} f_{i}^{(k-1)}+q_{i}^{-n+k+1} f_{i}^{(n-k-1)} f_{j} f_{i}^{(k)}\right) .
\end{aligned}
$$

It is easy to show that the second term is 0 .

Q.E.D.

\section{Lemma 2.1.9.}

$$
M=\operatorname{Ker} e_{i}^{\prime} .
$$

Proof. By Lemma 2.1.8, we have

$$
M \subset \operatorname{Ker} e_{i}^{\prime} .
$$

We recall the result in $[1]$,

$$
U_{q}^{-}=\operatorname{Ker} e_{i}^{\prime} \oplus f_{i}\left(U_{q}^{-}\right) .
$$

Then this lemma follows from Corollary 2.1.5.

Q.E.D.

Proof of Proposition 2.1.2. By Lemmas 2.1.6 and 2.1.9, we have $\operatorname{Ker} e_{i}^{\prime} \subset T_{2}\left(U_{q}^{-}\right) \cap U_{q}^{-}$.

Then this proposition follows from Lemma 2.1.3, (2.1.10) and (2.1.12).

Q.E.D.

\section{§ 3. Crystals}

\subsection{Definition of crystal}

Definition 3.1.1. A crystal $B$ is a set with

$$
\begin{gathered}
\text { maps wt }: B \rightarrow P, \varepsilon_{\imath}: B \rightarrow \boldsymbol{Z} \cup\{-\infty\} \text { and } \varphi_{\imath}: B \rightarrow \boldsymbol{Z} \cup\{-\infty\}, \\
\tilde{e}_{\imath}: B \longrightarrow B \cup\{0\}, \quad \tilde{f}_{i}: B \longrightarrow B \cup\{0\} .
\end{gathered}
$$

They are subject to the following axioms:

$$
\varphi_{\imath}(b)=\varepsilon_{i}(b)+\left\langle h_{\imath}, w t(b)\right\rangle .
$$

If $b \in B$ and $\tilde{e}_{i} b \in B$ then,

$$
w t\left(\tilde{e}_{i} b\right)=w t(b)+\alpha_{\imath}, \varepsilon_{\imath}\left(\tilde{e}_{\imath} b\right)=\varepsilon_{\imath}(b)-1 \text { and } \varphi_{\imath}\left(\tilde{e}_{i} b\right)=\varphi_{\imath}(b)+1 \text {. }
$$

If $b \in B$ and $\tilde{f}_{i} b \in B$, then $w t\left(\tilde{f}_{i} b\right)=w t(b)-\alpha_{i}, \varepsilon_{\imath}\left(\tilde{f}_{\imath} b\right)=\varepsilon_{\imath}(b)+1$ and $\varphi_{\imath}\left(\tilde{f}_{i} b\right)=\varphi_{\imath}(b)-1$.

(C3) For $b, b^{\prime} \in B$ and $i \in I, b^{\prime}=\tilde{e}_{2} b$ if and only if $b=\tilde{f}_{2} b^{\prime}$.

(C4) For $b \in B$, if $\varphi_{2}(b)=-\infty$, then $\tilde{e}_{2} b=\tilde{f}_{2} b=0$. 
For two crystals $B_{1}$ and $B_{2}$, a morphism $\psi$ from $B_{1}$ to $B_{2}$ is a map $B_{1} \rightarrow$ $B_{2} \cup\{0\}$ that satisfies the following conditions:

$$
\begin{aligned}
& \text { If } b \in B_{1} \text { and } \psi(b) \in B_{2} \text {, then } w t(\psi(b))=w t(b), \varepsilon_{\imath}(\phi(b)) \\
& =\varepsilon_{\imath}(b) \text {, and } \varphi_{\imath}(\psi(b))=\varphi_{\imath}(b) \text {, }
\end{aligned}
$$

$$
\text { For } b \in B_{1} \text {, we have } \psi\left(\tilde{e}_{2} b\right)=\tilde{e}_{2} \psi(b) \text { provided } \psi(b) \text { and } \psi\left(\tilde{e}_{i} b\right) \in B_{2} \text {, }
$$$$
\text { For } b \in B_{1} \text {, we have } \phi\left(\tilde{f}_{\imath} b\right)=\tilde{f}_{2} \psi(b) \text { provided } \psi(b) \text { and } \psi\left(\tilde{f}_{i} b\right) \in B_{2} \text {. }
$$

A morphism $\psi: B_{1} \rightarrow B_{2}$ is called strict, if it commutes with all $\tilde{e}_{2}$ and $\tilde{f}_{\imath}$.

A morphism $\phi: B_{1} \rightarrow B_{2}$ is called an embedding, if $\psi$ induces an injective map $B_{1} \cup\{0\} \rightarrow B_{2} \cup\{0\}$.

For two crystals $B_{1}$ and $B_{2}$, we define its tensor product $B_{1} \otimes B_{2}$ as follows:

$$
\begin{aligned}
& B_{1} \otimes B_{2}=\left\{b_{1} \otimes b_{2} ; b_{1} \in B_{1} \text { and } b_{2} \in B_{2}\right\} \\
& \varepsilon_{\imath}\left(b_{1} \otimes b_{2}\right)=\max \left\{\varepsilon_{i}\left(b_{1}\right), \varepsilon_{i}\left(b_{2}\right)-w t_{i}\left(b_{1}\right)\right\} \\
& \varphi_{\imath}\left(b_{1} \otimes b_{2}\right)=\max \left\{\varphi_{\imath}\left(b_{1}\right)+w t_{i}\left(b_{2}\right), \varphi_{\imath}\left(b_{2}\right)\right\} \\
& w t\left(b_{1} \otimes b_{2}\right)=w t\left(b_{1}\right)+w t\left(b_{2}\right) .
\end{aligned}
$$

Here $w t_{2}(b)=\left\langle h_{\imath}, w t(b)\right\rangle$. The action of $\tilde{e}_{i}$ and $\tilde{f}_{\imath}$ are defined by

$$
\begin{aligned}
& \tilde{e}_{2}\left(b_{1} \otimes b_{2}\right)=\left\{\begin{array}{lll}
\tilde{e}_{i} b_{1} \otimes b_{2} & \text { if } & \varphi_{\imath}\left(b_{1}\right) \geqq \varepsilon_{\imath}\left(b_{2}\right) \\
b_{1} \otimes \tilde{e}_{i} b_{2} & \text { if } & \varphi_{\imath}\left(b_{1}\right)<\varepsilon_{\imath}\left(b_{2}\right)
\end{array}\right. \\
& \tilde{f}_{i}\left(b_{1} \otimes b_{2}\right)=\left\{\begin{array}{lll}
\tilde{f}_{i} b_{1} \otimes b_{2} & \text { if } & \varphi_{i}\left(b_{1}\right)>\varepsilon_{i}\left(b_{2}\right) \\
b_{1} \otimes \tilde{f}_{i} b_{2} & \text { if } & \varphi_{i}\left(b_{1}\right) \leqq \varepsilon_{\imath}\left(b_{2}\right) .
\end{array}\right.
\end{aligned}
$$

Example 3.1.2. For $i \in I, B_{\imath}$ is the crystal defined as follows

$$
\begin{gathered}
B_{\imath}=\left\{b_{\imath}(n) ; n \in \boldsymbol{Z}\right\} \\
w t\left(b_{i}(n)\right)=n \\
\varphi_{i}\left(b_{i}(n)\right)=n, \quad \varepsilon_{i}\left(b_{i}(n)\right)=-n \\
\varphi_{\jmath}\left(b_{i}(n)\right)=\varepsilon_{\jmath}\left(b_{i}(n)\right)=-\infty \quad \text { for } i \neq j .
\end{gathered}
$$

We define the action of $\tilde{e}_{\imath}$ and $\tilde{f}_{i}$ by

$$
\begin{gathered}
\tilde{e}_{i}\left(b_{i}(n)\right)=b_{\imath}(n+1) \\
\tilde{f}_{i}\left(b_{i}(n)\right)=b_{i}(n-1) \\
\tilde{e}_{\jmath}\left(b_{\imath}(n)\right)=\tilde{f}_{\jmath}\left(b_{\imath}(n)\right)=0 \quad \text { for } \quad i \neq j .
\end{gathered}
$$


We write $b_{i}$ for $b_{i}(0)$.

Example 3.1.3. For $\lambda \in P_{+}, B(\lambda)$ is the crystal associated with the crystal base of the simple module with highest weight $\lambda$. The unique element of $B(\lambda)$ of weight $\lambda$ is denoted by $u_{\lambda}$.

Example 3.1.4. $B(\infty)$ is the crystal associated with the crystal base of $U_{\bar{q}}^{-}$. We set $\varepsilon_{i}(b)=\max \left\{k \geqq 0 ; \tilde{e}_{i}^{k} b \pm 0\right\}, \varphi_{\imath}(b)=\varepsilon_{\imath}(b)+\left\langle h_{\imath}, w t(b)\right\rangle$. The unique element of $B(\infty)$ of weight 0 is denoted by $u_{\infty}$.

\subsection{Some results}

Theorem 3.2. ([3]).

$$
B(\infty)^{*}=B(\infty)
$$

We define the operators $\tilde{e}_{\imath}^{*}, \tilde{f}_{2}^{*}$ of $U_{q}^{-}$by

$$
\tilde{e}_{i}^{*}=* \tilde{e}_{2} * \text {, and } \tilde{f}_{i}^{*}=* \tilde{f}_{i} * \text {. }
$$

Theorem 3.2.2 ([3]). 1. For any $i$, there exists a unique strict embedding of crystals

$$
\Psi_{i}: B(\infty) \subset B(\infty) \otimes B_{\imath}
$$

that sends $u_{\infty}$ to $u_{\infty} \otimes b_{\imath}$.

2. If $\Psi_{\imath}(b)=b^{\prime} \otimes \tilde{f}_{\imath}^{n} b_{\imath}(n \geqq 0)$, then $\varepsilon_{\imath}\left(b^{*}\right)=n, \varepsilon_{\imath}\left(b^{*}\right)=0$ and

$$
\begin{gathered}
\Psi_{\imath}\left(\tilde{e}_{\imath}^{*} b\right)=\left\{\begin{array}{lll}
b^{\prime} \otimes \tilde{f}_{\imath}^{n-1} b_{\imath} & \text { if } & n \geqq 0 \\
0 & \text { if } & n=0
\end{array}\right. \\
\Psi_{\imath}\left(\tilde{f}_{\imath}^{*} b\right)=b^{\prime} \otimes \tilde{f}_{\imath}^{n+1} b_{\imath}
\end{gathered}
$$

3. Im $\Psi_{i}=\left\{b \otimes \tilde{f}_{\imath}^{n} b_{\imath}: b \in B(\infty), \varepsilon_{\imath}\left(b^{*}\right)=0, n \geqq 0\right\}$.

\subsection{Action on $L(\lambda)$}

Lemma 3.3.1 ([3]). For $b \in B(\infty)$

$$
\begin{aligned}
& f_{i}^{(a)} G(b) \equiv\left[\begin{array}{c}
\varepsilon_{\imath}(b)+a \\
a
\end{array}\right]_{i} G\left(\tilde{f}_{i}^{a} b\right) \bmod f_{\imath}^{a+1} U_{q}^{-} \\
& G(b) f_{i}^{(a)} \equiv\left[\begin{array}{c}
\varepsilon_{\imath}\left(b^{*}\right)+a \\
a
\end{array}\right]_{i} G\left(\tilde{f}_{i}^{* a} b\right) \bmod U_{q}^{-} f_{i}^{a+1} .
\end{aligned}
$$

Let $\lambda \in P_{+}$, and let $V(\lambda)$ be the irreducible $U_{q}$-module generated by the highest weight vector $u_{\lambda}$ of highest weight $\lambda$. For $w \in W$, let us denote by $u_{w \lambda}$ the global base of weight $w \lambda$. Then we have 


$$
u_{w \lambda}=u_{\lambda} \quad \text { if } \quad w=1 .
$$

If $w=s_{i} w^{\prime}>w^{\prime}$, then we have

$$
u_{w \lambda}=f_{i}^{(c)} u_{w^{\prime} \lambda} \quad \text { where } c=\left\langle h_{\imath}, w \lambda\right\rangle .
$$

Lemma 3.3.2. For $b \in B(\infty)$

$$
G(b) u_{w \lambda}=G\left(\tilde{f}_{i}^{* c} b\right) u_{w^{\prime \lambda}} .
$$

Proof. Note that $f_{\imath} u_{w \lambda}=0$ and $f_{i}^{1+c} u_{w_{\lambda} \lambda}=0$. By Lemma 3.3.1,

$$
\begin{aligned}
G(b) u_{w \lambda} & =G(b) f_{i}^{(c)} u_{w^{\prime \lambda}} \\
& =\left(\left[\begin{array}{c}
\varepsilon_{\imath}\left(b^{*}\right)+c \\
c
\end{array}\right]_{i} G\left(\tilde{f}_{i}^{* c} b\right)+U_{q}^{-} f_{i}^{c+1}\right) u_{w^{\prime} \lambda} \\
& =\left[\begin{array}{c}
\varepsilon_{i}\left(b^{*}\right)+c \\
c
\end{array}\right]_{i} G\left(\tilde{f}_{i}^{* c} b\right) u_{w^{\prime} \lambda} .
\end{aligned}
$$

If $\varepsilon_{i}\left(b^{*}\right)=0$, it is obvious. If $\varepsilon_{i}\left(b^{*}\right) \neq 0$, then we have

$$
G(b) u_{w \lambda} \in U_{q}^{-} f_{i} u_{w \lambda}=0
$$

and

$$
G\left(\tilde{f}_{i}^{* c} b\right) u_{w^{\prime} \lambda} \in U_{q}^{-} f_{i}^{c+1} u_{w^{\prime} \lambda}=0
$$

since $G(b) \in U_{q}^{-} f_{i}^{\varepsilon_{i}(b *)}$ and $\varepsilon_{i}\left(\left(\tilde{f}_{i}^{* c} b\right)^{*}\right)=\varepsilon_{i}\left(b^{*}\right)+c$.

Corollary 3.3.3. Let $\lambda \in P_{+}, P \in L(\infty)$ and $b \in B(\infty)$ such that $b \equiv P \bmod q L(\infty)$. Then we have

$$
P u_{s_{i} \lambda} \equiv G\left(\tilde{f}_{i}^{*\left\langle h_{i}, \lambda\right\rangle} b\right) u_{\lambda} \bmod q L(\lambda) .
$$

\section{Proposition 3.3.4.}

$$
T_{i}(P) u_{\lambda} \in L(\lambda)
$$

and

$$
T_{\imath}(P) u_{\lambda} \equiv \begin{cases}\tilde{e}_{i}^{m} G\left(\tilde{f}_{i}^{*\left\langle h_{i}, \lambda\right\rangle} b\right) u_{\lambda} & \text { if } \varphi\left(\tilde{f}_{i}^{*\left\langle h_{i}, \lambda\right\rangle} b\right)+\left\langle h_{i}, \lambda\right\rangle=0 \\ 0 & \text { if } \varphi_{i}\left(\tilde{f}_{i}^{*\left\langle h_{i}, \lambda\right\rangle} b\right)+\left\langle h_{i}, \lambda\right\rangle \neq 0\end{cases}
$$

where $\left.m=\varepsilon_{i}\left(\tilde{f}_{i}^{*\left\langle h_{i}\right.}, \lambda\right\rangle b\right)$.

Proof. We recall (1.2.4)

$$
S_{i} u_{k}^{(l)}=(-1)^{l-k} q_{i}^{(l-k)(k+1)} u_{l-k}^{(l)} .
$$

So, $L(\lambda)$ and $L(\lambda) / q L(\lambda)$ are stable by $S_{i}$, and for $b \in B(\lambda)$ 


$$
S_{\imath} b=\left\{\begin{array}{lll}
\tilde{e}_{i}^{\varepsilon_{i}(b)} b & \text { if } & \varphi_{i}(b)=0 \\
0 & \text { if } & \varphi_{i}(b) \neq 0
\end{array}\right.
$$

In particular,

$$
S_{i} P u_{s_{i} \lambda} \equiv\left\{\begin{array}{lll}
\tilde{e}_{i}^{m} G\left(\tilde{f}_{i}^{*\left\langle h_{i}, \lambda\right\rangle} b\right) u_{\lambda} & \text { if } \quad \varphi_{i}\left(\tilde{f}_{i}^{*\left\langle h_{i}, \lambda\right\rangle} b\right)+\left\langle h_{i}, \lambda\right\rangle=0 \\
0 & \text { if } \quad \varphi_{i}\left(\tilde{f}_{i}^{*\left\langle h_{i}, \lambda\right\rangle} b\right)+\left\langle h_{i}, \lambda\right\rangle \neq 0 .
\end{array}\right.
$$

On the other hand, by (3.3.10)

$$
\begin{aligned}
S_{\imath} P u_{s_{i} \lambda} & =T_{\imath}(P) S_{\imath} u_{s_{i} \lambda} \\
& =T_{\imath}(P) u_{\lambda} .
\end{aligned}
$$

Therefore, we have (3.3.8) and (3.3.9).

Q.E.D.

\subsection{Action on $L(\infty)$}

Let $b \in B(\infty)$ and $\lambda \in P_{+}$.

Lemma 3.4.1. $G(b) u_{\lambda} \neq 0$ if and only if $\varepsilon_{i}\left(b^{*}\right) \leqq\left\langle h_{i}, \lambda\right\rangle$ for any $i$.

Proof.

$$
\left\{P \in U_{q}^{-} ; P u_{\lambda}=0\right\}=\sum_{i} U_{q}^{-} f_{i}^{1+\left\langle h_{i}, \lambda\right\rangle}
$$

and

$$
U_{q}^{-} f_{\imath}^{a}=\underset{\varepsilon_{i}(b *) \geqq a}{\bigoplus} \boldsymbol{Q}(q) G(b) .
$$

So, we have

$$
\left\{P \in U_{q}^{-} ; P u_{\lambda}=0\right\}=\sum_{i} \bigoplus_{\varepsilon_{i}(b *)>\left\langle h_{i}, \lambda\right\rangle} \boldsymbol{Q}(q) G(b) .
$$

Lemma 3.4.2. ([2]).

$$
\varepsilon_{\jmath}\left(\tilde{f}_{i}^{a} b\right) \leqq \varepsilon_{\jmath}(b) \quad \text { for } \quad i \neq j .
$$

Now, let us assume that $\left\langle h_{\imath}, \lambda\right\rangle$ is sufficiently large for any $i$.

Lemma 3.4.3. If $\varepsilon_{i}\left(b^{*}\right)=0$, then $G\left(\tilde{f}_{i}^{*\left\langle h_{i}, \lambda\right\rangle} b\right) u_{\lambda} \neq 0$.

Proof. By Lemma 3.4.1, $G\left(\tilde{f}_{i}^{*\left\langle h_{i}, \lambda\right\rangle} b\right) u_{\lambda} \neq 0$ if and only if $\varepsilon_{j}^{*}\left(\tilde{f}_{i}^{*\left\langle h_{i}, \lambda\right\rangle} b\right) \leqq$ $\left\langle h_{j}, \lambda\right\rangle$ for any $j$, where we set $\varepsilon_{i}^{*}(b)=\varepsilon_{\imath}\left(b^{*}\right)$ for $b \in B(\infty)$. If $i=j$, then

If $i \neq j$, then

$$
\varepsilon_{j}^{*}\left(\tilde{f}_{i}^{*\left\langle h_{i}, \lambda\right\rangle} b\right)=\left\langle h_{i}, \lambda\right\rangle+\varepsilon_{i}^{*}(b)=\left\langle h_{i}, \lambda\right\rangle .
$$

since $\lambda \gg 0$.

$$
\varepsilon_{j}^{*}\left(\tilde{f}_{i}^{*\left\langle h_{i}, \lambda\right\rangle} b\right) \leqq \varepsilon_{j}^{*}(b) \leqq \lambda_{j}
$$

Let $b$ be an element of $B(\infty)$ and let us assume that $P=G(b)$ belongs to $T_{i}^{-1} U_{q}^{-} \cap U_{q}^{-}$. 
Lemma 3.4.4.

$$
\left.G\left(\tilde{f}_{i}^{*\left\langle h_{i}\right.}, \lambda\right\rangle b\right) u_{\lambda} \neq 0 \text {. }
$$

Proof. Since $T_{\imath}^{-1}=* T_{\imath} *$ and $U_{q}^{-}$is stable under *

$$
\begin{aligned}
T_{i}^{-1}\left(U_{\bar{q}}^{-}\right) \cap U_{q}^{-} & =\left(T_{\imath}\left(U_{q}^{-}\right) \cap U_{q}^{-}\right)^{*} \\
& =\left(\operatorname{Ker} e_{i}^{\prime}\right)^{*} .
\end{aligned}
$$

Then we have $e_{i}^{\prime} P^{*}=0$, and therefore $\tilde{e}_{i} P^{*}=0$. This implies

$$
\varepsilon_{i}^{*}(b)=0 \text {. }
$$

By Lemma 3.4.3, we have (3.4.2).

Q.E. D.

\section{Lemma 3.4.5.}

$$
T_{\imath}(P) u_{\lambda} \neq 0
$$

Proof. By Proposition 3.3.4, it is enough to show that $\varphi_{2}\left(\tilde{f}_{i}^{*\left\langle h_{i}, \lambda\right\rangle} b\right)+\left\langle h_{i}, \lambda\right\rangle$ $=0$. We have

$$
\varphi_{i}\left(\tilde{f}_{i}^{*\left\langle h_{i}, \lambda\right\rangle} b\right)+\left\langle h_{i}, \lambda\right\rangle=\varepsilon_{i}\left(\tilde{f}_{i}^{*\left\langle h_{i}, \lambda\right\rangle} b\right)+\left\langle h_{i}, w t(b)-\lambda\right\rangle .
$$

Since $\varepsilon_{i}^{*}(b)=0, \Psi_{i}(b)=b \otimes b_{i}$ and $\Psi_{i}\left(\tilde{f}_{i}^{*\left\langle h_{i}, \lambda\right\rangle} b\right)=b \otimes \tilde{f}_{i}^{\left\langle h_{i}, \lambda\right\rangle} b_{\iota}$,

$$
\begin{aligned}
\varepsilon_{i}\left(\tilde{f}_{i}^{*\left\langle h_{i}, \lambda\right\rangle} b\right) & =\varepsilon_{i}\left(b \otimes \tilde{f}_{i}^{\left\langle h_{i}, \lambda\right\rangle} b_{i}\right) \\
& =\max \left\{\varepsilon_{i}(b), \varepsilon_{i}\left(\tilde{f}_{i}^{*\left\langle h_{i}, \lambda\right\rangle} b_{i}\right)-\left\langle h_{i}, w t(b)\right\rangle\right\} \\
& =\max \left\{\varepsilon_{i}(b),\left\langle h_{i}, \lambda\right\rangle-\left\langle h_{i}, w t(b)\right\rangle\right\} .
\end{aligned}
$$

Therefore we have

$$
\varepsilon_{i}\left(\tilde{f}_{i}^{*\left\langle h_{i}, \lambda\right\rangle} b\right)=\left\langle h_{i}, \lambda-w t(b)\right\rangle .
$$

Since $\left\langle h_{i}, \lambda\right\rangle$ is sufficently large, it equals $\left\langle h_{\imath}, \lambda-w t(b)\right\rangle$. Therefore $\varphi_{i}\left(\tilde{f}_{i}^{*\left\langle h_{i}, \lambda\right\rangle} b\right)$ $+\left\langle h_{i}, \lambda\right\rangle=0$.

Q.E.D.

\section{Lemma 3.4.6.}

$$
\varphi_{\imath}(b)+\varepsilon_{\imath}^{*}(b) \geqq 0 \quad \text { for any } b \in B(\infty) .
$$

Proof. By Theorem 3.2.2

$$
\Psi_{i}(b)=b^{\prime} \otimes \tilde{f}_{i}^{n} b_{\imath}
$$

where $n=\varepsilon_{i}^{*}(b)=-\varphi_{i}\left(\tilde{f}_{i}^{n} b_{i}\right)$. We have

$$
\begin{aligned}
\varphi_{i}(b) & =\varphi_{i}\left(b^{\prime} \otimes \tilde{f}_{i}^{n} b_{i}\right) \\
& =\max \left\{\varphi_{i}\left(b^{\prime}\right)+\left\langle h_{i}, w t\left(\tilde{f}_{i}^{n} b_{i}\right)\right\rangle, \varphi_{i}\left(\tilde{f}_{i}^{n} b_{i}\right)\right\} .
\end{aligned}
$$


Therefore we have

$$
\varphi_{\imath}(b)+\varepsilon_{\imath}^{*}(b)=\varphi_{\imath}(b)-\varphi_{\imath}\left(\tilde{f}_{\imath}^{n} b_{\imath}\right) \geqq 0 .
$$

Q.E.D.

\section{Proposition 3.4.7.}

$$
\begin{gathered}
T_{\imath} P \in L(\infty) . \\
T_{\imath} P \equiv \tilde{f}_{\imath}^{* \varphi_{\imath}(b)} \tilde{e}_{\imath}^{\varepsilon_{\imath}(b)} b \bmod q L(\infty) .
\end{gathered}
$$

Proof. By Lemma 3.4.6, we have

$$
\varphi_{\imath}(b)=\varphi_{\imath}(b)+\varepsilon_{\imath}^{*}(b) \geqq 0 .
$$

By (3.4.3), (3.4.6) and (3.4.10), we have

$$
\begin{aligned}
\Psi_{\imath}\left(\tilde{e}_{i}^{m} \tilde{f}_{\imath}^{*\left\langle h_{\imath}, \lambda\right\rangle} b\right) & =\tilde{e}_{\imath}^{\left\langle h_{\imath}, \lambda-w \iota(b)\right\rangle}\left(b \otimes \tilde{f}_{\imath}^{\left\langle h_{\imath}, \lambda\right\rangle} b_{\imath}\right) \\
& =\tilde{e}_{\imath}^{\varepsilon_{\imath}(b)} b \otimes \tilde{f}_{\imath}^{\varphi_{\imath}(b)} b_{\imath} .
\end{aligned}
$$

On the other hand, by Theorem 3.2.2 we have

$$
\begin{aligned}
\Psi_{\imath}\left(\tilde{f}_{i}^{* \varphi_{i}(b)} \tilde{e}_{\imath}^{\varepsilon_{2}(b)} b\right) & =\tilde{f}_{i}^{* \varphi_{\imath}(b)}\left(\tilde{e}_{\imath}^{\varepsilon_{\imath}(b)} b \otimes b_{\imath}\right) \\
& =\tilde{e}_{\imath}^{\varepsilon_{\imath}(b)} b \otimes \tilde{f}_{\imath}^{\varphi_{\imath}(b)} b_{\imath} .
\end{aligned}
$$

Since $\Psi_{\imath}$ is an embedding, we have

$$
\tilde{e}_{\imath}^{m} \tilde{f}_{\imath}^{*\left\langle h_{\imath}, \lambda\right\rangle} b=\tilde{f}_{\imath}^{* \varphi_{\imath}(b)} \tilde{e}_{\imath}^{\varepsilon_{\imath}(b)} b .
$$

Therefore we obtain

$$
T_{\imath}(P) u_{\lambda} \equiv G\left(\tilde{f}_{\imath}^{* \varphi_{\imath}(b)} \tilde{e}_{\imath}^{\varepsilon_{i}(b)} b\right) u_{\lambda} .
$$

For $\lambda \gg 0$, Proposition 3.3.4 and (3.4.12) imply this proposition.

Q.E.D.

Corollary 3.4.8. Let us define the map $\Lambda_{\imath}:\left\{b \in B(\infty) ; \varepsilon_{i}^{*}(b)=0\right\} \rightarrow\{b \in B(\infty)$; $\left.\varepsilon_{\imath}(b)=0\right\}$ by $b \mapsto \tilde{f}_{i}^{* \varphi_{\imath}(b)} \tilde{e}_{i}^{\varepsilon_{i}(b)} b$. Then $\Lambda_{2}$ is bijective and $\Lambda_{\imath}^{-1}(b)=\tilde{f}_{i}^{\varphi_{i}^{*}(b)} \tilde{e}_{2}^{* \varepsilon_{i}^{*}(b)} b$.

Proof. Let $b \in\left\{b \in B(\infty) ; \varepsilon_{\imath}^{*}(b)=0\right\}, b^{\prime}=\tilde{f}_{\imath}^{* \varphi_{\imath}(b)} \tilde{e}_{\imath}^{\varepsilon_{i}{ }^{(b)}} b . \quad$ By Proposition 3.4.7 $\varepsilon_{\imath}\left(b^{\prime}\right)=0$. We have

$$
\varepsilon_{\imath}^{*}\left(b^{\prime}\right)=\varphi_{\imath}(b) .
$$

Indeed we have $\Psi_{\iota}\left(b^{\prime}\right)=\tilde{e}_{\imath_{\imath}}^{\varepsilon^{(b)}} b \otimes \tilde{f}_{\imath^{(}{ }^{(b)}} b_{\imath}$. Theorem 3.2.2 implies (3.4.13). We get

$$
\begin{aligned}
\varphi_{\imath}^{*}\left(b^{\prime}\right) & =\varepsilon_{i}^{*}\left(b^{\prime}\right)+\left\langle h_{\imath}, w t\left(b^{\prime}\right)\right\rangle \\
& =\varphi_{\imath}(b)+\left\langle h_{\imath},\left(\varepsilon_{\imath}(b)-\varphi_{\imath}(b)\right) \alpha_{\imath}+w t(b)\right\rangle \\
& =2 \varepsilon_{\imath}(b)-\varphi_{\imath}(b)+\left\langle h_{\imath}, w t(b)\right\rangle \\
& =\varepsilon_{\imath}(b) .
\end{aligned}
$$


By Theorem 3.2.2 and (3.4.13), we have

Therefore we obtain

$$
\begin{aligned}
\Psi_{\imath}\left(\tilde{e}_{i}^{* \varepsilon_{i}^{*}\left(b^{\prime}\right)} b^{\prime}\right) & =\tilde{e}_{i}^{\varepsilon_{i}(b)} b \otimes b_{\imath} \\
& =\Psi_{i}\left(\tilde{e}_{i}^{\varepsilon_{i}(b)} b\right) .
\end{aligned}
$$

Hence, we have

$$
\tilde{e}_{i}^{* \varepsilon_{i}^{*}\left(b^{\prime}\right)} b^{\prime}=\tilde{e}_{i}^{\varepsilon_{i}(b)} b
$$

$$
\tilde{f}_{i}^{\varphi *}{ }_{i}^{*}\left(b^{\prime}\right) \tilde{e}_{i}^{* \varepsilon_{i}^{*}\left(b^{\prime}\right)} b^{\prime}=\tilde{f}_{i}^{\varepsilon_{i}^{(b)}} \tilde{e}_{i}^{\varepsilon^{(b)}} b=b .
$$

Let $b \in\left\{b \in B(\infty) ; \varepsilon_{\imath}(b)=0\right\}$ and $b^{\prime}=\tilde{f}_{\imath}^{\varphi_{i}^{*}(b)} \tilde{e}_{\imath}^{* \varepsilon_{\imath}^{*}(b)} b$. We have the following formulas similarly

$$
\begin{gathered}
\varepsilon_{i}^{*}\left(b^{\prime}\right)=0 \\
\tilde{f}_{\imath}^{*\left(\rho_{i}(b)\right.} \tilde{e}_{\imath}^{\varepsilon_{i}(b)} b^{\prime}=b .
\end{gathered}
$$

The corollary is proved.

Q.E.D.

\section{§4. Main Theorem}

\subsection{Proof of Main Theorem}

In this section, we assume that $g$ is a finite-dimensional semisimple Lie algebra.

Proposition 4.1.1 [4]. (1) Let $w \in W$ and let $s_{\imath_{1}} \cdots s_{\imath_{k}}$ be a reduced expression of $w$. Then the automorphism $T_{w}=T_{\imath_{1}} \cdots T_{\imath_{k}}$ of $U_{q}$ is independent of the choice of the reduced expression of $w$.

(2) If $w \alpha_{i} \in R^{+}$, then $T_{w} f_{\imath} \in U_{q}^{-}$.

Fix a reduced expression $s_{\imath_{1}} \cdots s_{\imath_{N}}$ of the longest element of $W$. This gives us an ordering of the set of all positive roots $R^{+}$

$$
\beta_{1}=\alpha_{\imath_{1}}, \quad \beta_{2}=s_{\imath_{1}} \alpha_{\imath_{2}}, \cdots, \quad \beta_{N}=s_{\imath_{1}} \cdots s_{\imath_{N-1}} \alpha_{i_{N}} .
$$

We define

$$
f_{\beta_{m}}=T_{\imath_{1}} \cdots T_{\imath_{m-1}}\left(f_{i_{m}}\right)
$$

and

$$
f^{k}=f_{\beta_{1}}^{\left(k_{1}\right)} f_{\beta_{2}}^{\left(k_{2}\right)} \cdots f_{\beta N}^{(k N)} \quad \text { where } \quad k=\left(k_{1}, \cdots, k_{N}\right) \in \boldsymbol{Z}_{\geqq 0}^{N}
$$

\section{Theorem 4.1.2 (Main Theorem).}

$$
f^{k} \in L(\infty) \quad \text { for any } k=\left(k_{1}, \cdots, k_{N}\right) \in \boldsymbol{Z}_{\geq 0}^{N} .
$$

$$
\left\{f^{k} \bmod q L(\infty) ; k \in \boldsymbol{Z}_{\geqq 0}^{N}\right\}=B(\infty) .
$$

Proof. Let $P \in L(\infty)$ such that $T_{\imath} P \in U_{\bar{q}}^{-}$. Then we have $e_{i}^{\prime} T_{i} P=0$. 
Therefore

$$
f_{i}^{(n)} T_{\imath} P=\tilde{f}_{\imath}^{n} T_{\imath} P
$$

Hence we obtain

$$
f_{i}^{(n)} T_{\imath} P \in L(\infty)
$$

and

$$
f_{i}^{(n)} T_{\imath} P \equiv \tilde{f}_{\imath}^{n} \tilde{f}_{\imath}^{* \varphi_{\imath}(b)} \tilde{e}_{\imath}^{\varepsilon_{\imath}(b)} b \bmod q L(\infty) \text { if } P \equiv b \bmod q L(\infty) .
$$

By (4.1.5), we have $f^{k} \in L(\infty)$ immediately.

By Proposition 3.4.7, we have $f^{k} \bmod q L(\infty) \in B(\infty)$ for any $k$. So, there exist the cannonical map $\pi:\left\{f^{k}\right\} \rightarrow B(\infty)$ by $f^{k} \mapsto f^{k} \bmod q L(\infty)$. We write $b^{k}$ for $f^{k} \bmod q L(\infty)$.

The first step is the next lemma.

Lemma 4.1.3. $\pi$ is injective.

Proof. Let $b_{(1)}=T_{\imath_{1}} f_{\imath_{2}}^{\left(k_{2}\right)} \cdots T_{\imath_{N-1}} f_{i_{N}}^{\left(k_{N}\right)} \bmod q L(\infty) \in B(\infty)$. Then we have

and

$$
b^{k}=\tilde{f}_{i_{1}^{1}}^{k_{1}} b_{(1)}
$$

$$
T_{\imath_{1}} f_{\left.i_{2}\right)}^{\left(k_{2}\right)} \cdots T_{\imath_{N-1}} f_{\imath_{N}}^{\left(k_{N}\right)} \in T_{\imath_{1}}\left(U_{q}^{-}\right) \cap U_{q}^{-}=\operatorname{Ker} e_{\imath_{-1}}^{\prime} .
$$

Therefore we have $\tilde{e}_{\imath_{1}} b_{(1)}=0$. Hence we obtain $k_{1}=\varepsilon_{\imath_{1}}\left(b^{k}\right)$. This implies

$$
b_{(1)}=\tilde{e}_{i_{1} i^{i_{1}}}^{\left(b^{k}\right)} b^{k} \text {. }
$$

By Corollary 3.4.8, we have

$$
f_{i_{2}}^{\left(k_{2}\right)} T_{\imath_{3}} \cdots T_{\iota_{N-1}} f_{i_{N}}^{\left(k_{N}\right)} \equiv \Lambda_{i_{1}}^{-1}\left(b_{(1)}\right) \bmod q L(\infty) .
$$

Let $b_{(2)}=\tilde{e}_{2_{2}}^{\varepsilon_{2}\left(1_{1} i_{1}^{-1}(b(1))\right)} \Lambda_{i_{1}}^{-1}\left(b_{1}\right)$. Then similarly we have

and

$$
\begin{gathered}
\varepsilon_{\imath_{2}}\left(\Lambda_{i_{1}}^{-1}\left(b_{2}\right)\right)=k_{2}, \\
b_{(2)} \equiv T_{\imath_{2}} f_{i_{3}}^{\left(k_{3}\right)} \ldots T_{{ }{ }_{N-1}} f_{i_{N}}^{\left(k_{N}\right)},
\end{gathered}
$$

$$
f_{i_{3}}^{\left(k_{3}\right)} \cdots T_{\imath_{N-1}} f_{i_{N}}^{\left(k_{N}\right)} \equiv \Lambda_{i_{2}}^{-1}\left(b_{(2)}\right) \bmod q L(\infty) .
$$

Repeating this, $k=\left(k_{1}, \cdots, k_{N}\right)$ is uniquly determined by $b^{k}$.

Now we define a map $b^{k} \mapsto f^{\rho(b k)}$. It is trivial that this map is $\pi^{-1}$. Therefore $\pi$ is injective.

Q.E.D.

Let $Q_{-}=\Sigma \boldsymbol{Z}_{\leqq 0} \alpha_{\imath}$.

$$
t_{i} f_{\beta_{m}} t_{i}^{-1}=q_{i}^{-\left\langle h_{\imath}, \beta_{m}\right\rangle} f_{\beta_{m}} .
$$

For $\xi \in Q_{-}$, we set 


$$
B_{\xi}=\left\{b^{k} ; k \in \mathbb{Z}_{\geq 0}^{N}, w t\left(b^{k}\right)=\xi\right\} .
$$

Hence we have $B_{\xi} \subseteq B(\infty)_{\xi}$. By (4.1.7), we obtain

$$
\# B_{\xi}=\#\left\{\left(c_{1}, \cdots, c_{N}\right) \in \mathbb{Z}_{\geqq 0}^{N} ; \xi=-\sum c_{i} \beta_{\imath}\right\} .
$$

On the other hand, the PBW theorem for finite-dimensional semisimple Lie algebra implies

$$
\begin{aligned}
\# B(\infty)_{\xi} & =\operatorname{dim}_{Q(q)}\left(U_{q}^{-}\right)_{\xi} \\
& =\operatorname{dim}_{C}\left(U^{-}\right)_{\xi} \\
& =\#\left\{\left(c_{1}, \cdots, c_{N}\right) \in Z_{\geqq 0}^{N} ; \xi=-\sum c_{\imath} \beta_{\imath}\right\} .
\end{aligned}
$$

Therefore

Hence we obtain (ii).

$$
B_{\xi}=B(\infty)_{\xi}
$$

Q.E.D.

\subsection{Examples}

\section{Example 4.2.1.}

$$
\begin{gathered}
\mathrm{g}=A_{2}, \quad I=\{1,2\}, \quad a_{\imath \jmath}=a_{\jmath \imath}=-1, \\
\beta_{1}=\alpha_{1}, \quad \beta_{2}=\alpha_{1}+\alpha_{2}, \quad \beta_{3}=\alpha_{2} .
\end{gathered}
$$

In this case, $\Psi_{121}: B(\infty) \subset u_{\infty} \otimes B_{1} \otimes B_{2} \otimes B_{1}$. We shall calculate $\Psi_{121}\left(f^{\left(k_{1} k_{2} k_{3}\right)}\right)$. First we have

$$
\begin{gathered}
\Psi_{1}\left(f_{1}^{\left(k_{3}\right)}\right)=u_{\infty} \otimes \tilde{f}_{1}^{k_{3}} b_{1}, \\
T_{2} f_{1}^{\left(k_{3}\right)} \equiv \tilde{f}_{2}^{* \varphi_{2}(b)} \tilde{e}_{2}^{\varepsilon_{2}^{(b)}} b \quad \text { where } \quad f_{1}^{\left(k_{3}\right)} \equiv b \bmod q L(\infty)
\end{gathered}
$$

and

$$
\varphi_{2}(b)=k_{3}, \quad \varepsilon_{2}(b)=0 .
$$

Therefore we have

$$
\begin{aligned}
T_{2} f_{1}^{\left(k_{3}\right)} \equiv & \tilde{f}_{2}^{* k_{3}} b \\
& \stackrel{\Psi_{2}}{\longrightarrow} b \otimes \tilde{f}_{2}^{k_{3}} b_{2} \\
& \stackrel{\Psi_{1}}{\longmapsto} u_{\infty} \otimes \tilde{f}_{1}^{k_{3}} b_{1} \otimes \tilde{f}_{2}^{k_{3}} b_{2} .
\end{aligned}
$$

Since $\varphi_{2}\left(u_{\infty} \otimes \tilde{f}_{1}^{k_{3}} b_{1}\right)=k_{3}$ and $\varepsilon_{2}\left(\tilde{f}_{1}^{k_{3}} b_{2}\right)=k_{3}$, we have

$$
\begin{aligned}
& f_{2}^{\left(k_{2}\right)} T_{2} f_{1}^{\left(k_{3}\right)} \longmapsto \tilde{f}_{2}^{k_{2}}\left(u_{\infty} \otimes \tilde{f}_{1}^{k_{3}} b_{1} \otimes \tilde{f}_{2}^{k_{3}} b_{2}\right) \\
&=u_{\infty} \otimes \tilde{f}_{1}^{k_{3}} b_{1} \otimes \tilde{f}_{2}^{k_{2}+k_{3}} b_{2} . \\
& T_{1} f_{2}^{\left(k_{2}\right)} T_{2} f_{1}^{\left(k_{3}\right)} \equiv \tilde{f}_{1}^{*\left(\varphi_{1}\left(b^{\prime}\right)\right.} \tilde{e}_{1}^{\varepsilon_{1}\left(b^{\prime}\right)} b, \quad \text { where } \quad b^{\prime} \equiv f_{2}^{\left(k_{2}\right)} T_{2} f_{1}^{\left(k_{3}\right)} .
\end{aligned}
$$

Since $\varphi_{1}\left(b^{\prime}\right)=\varphi_{1}\left(u_{\infty} \otimes \tilde{f}_{1}^{k_{3}} b_{1} \otimes \tilde{f}_{2}^{k_{2}+k_{3}} b_{2}\right)=k_{2}$ and $\varepsilon_{1}\left(b^{\prime}\right)=k_{3}$ we have 


$$
\begin{aligned}
T_{1} b^{\prime} & \longmapsto \tilde{e}_{1}^{\varepsilon_{1}\left(b^{\prime}\right)} b^{\prime} \otimes \tilde{f}_{1}^{k_{2}} b_{1} \\
& \longmapsto \tilde{e}_{1}^{k_{3}}\left(u_{\infty} \otimes \tilde{f}_{1}^{k_{3}} b_{1} \otimes \tilde{f}_{2}^{k_{2}+k_{3}} b_{2}\right) \otimes \tilde{f}_{1}^{k_{2}} b_{1} \\
& =u_{\infty} \otimes b_{1} \otimes \tilde{f}_{2}^{k_{2}+k_{3}} b_{2} \otimes \tilde{f}_{1}^{k_{1}} b_{1}
\end{aligned}
$$

Therefore we obtain

$$
\begin{aligned}
f^{\left(k_{1}, k_{2}, k_{3}\right)} & =f_{\beta_{1}}^{\left(k_{1}\right)} f_{\beta_{2}}^{\left(k_{2}\right)} f_{\beta_{3}}^{\left(k_{3}\right)} \\
& =f_{1}^{\left(k_{1}\right)} T_{1} f_{2}^{\left(k_{2}\right)} T_{2} f_{1}^{\left(k_{3}\right)} \\
& \equiv f_{1}^{\left(k_{1}\right)} T_{1} b^{\prime} \\
& \longmapsto \tilde{f}_{1}^{k_{1}}\left(u_{\infty} \otimes b_{1} \otimes \tilde{f}_{2}^{k_{2}+k_{3}} b_{2} \otimes \tilde{f}_{1}^{k_{2}} b_{1}\right) \\
& = \begin{cases}u_{\infty} \otimes \tilde{f}_{1}^{k_{1}} b_{1} \otimes \tilde{f}_{2}^{k_{2}+k_{3}} b_{2} \otimes \tilde{f}_{1}^{k_{2}} b_{1} & \left(k_{1}<k_{3}\right) \\
u_{\infty} \otimes \tilde{f}_{1}^{k_{3}} b_{1} \otimes \tilde{f}_{2}^{k_{2}+k_{3}} b_{2} \otimes \tilde{f}_{1}^{k_{1}+k_{2}-k_{3}} b_{1} & \left(k_{1} \geqq k_{3}\right) .\end{cases}
\end{aligned}
$$

By [3], we know

$$
B(\infty)=\left\{u_{\infty} \otimes \tilde{f}_{1}^{k_{2}} b_{1} \otimes \tilde{f}_{2}^{k_{2}+k_{3}} b_{2} \otimes \tilde{f}_{1}^{k_{1}} b_{1} ; 0 \leqq k_{1}, 0 \leqq k_{2}, 0 \leqq k_{3}\right\} .
$$

We shall calculate $\left(u_{\infty} \otimes \tilde{f}_{1}^{k_{2}} b_{1} \otimes \tilde{f}_{2}^{k_{2}+k_{3}} b_{2} \otimes \tilde{f}_{1}^{k_{1}} b_{1}\right)^{*}$. First we have

$$
\left(\tilde{f}_{1}^{k_{1}} \tilde{f}_{2}^{k_{2}+k_{3}} \tilde{f}_{1}^{k_{2}} u_{\infty}\right)^{*} \longmapsto u_{\infty} \otimes \tilde{f}_{1}^{k_{2}} b_{1} \otimes \tilde{f}_{2}^{k_{2}+k_{3}} b_{2} \otimes \tilde{f}_{1}^{k_{1}} b_{1} \text {. }
$$

And we have

$$
\begin{aligned}
& \tilde{f}_{1}^{k_{1}} \tilde{f}_{2}^{k_{2}+k_{3}} \tilde{f}_{1}^{k_{2}} u_{\infty} \longmapsto \tilde{f}_{1}^{k_{1}} \tilde{f}_{2}^{k_{2}+k_{3}}\left(u_{\infty} \otimes \tilde{f}_{1}^{k_{2}} b_{1}\right) \\
& \\
& \qquad \tilde{f}_{1}^{k_{1}}\left(u_{\infty} \otimes \tilde{f}_{2}^{k_{2}+k_{3}} b_{2} \otimes \tilde{f}_{1}^{k_{2}} b_{1}\right) \\
& \longmapsto \begin{cases}u_{\infty} \otimes \tilde{f}_{1}^{k_{1}} b_{1} \otimes \tilde{f}_{2}^{k_{2}+k_{3}} b_{2} \otimes \tilde{f}_{1}^{k_{2}} b_{1} & \left(k_{1} \leqq k_{3}\right) \\
u_{\infty} \otimes \tilde{f}_{1}^{k_{3}} b_{1} \otimes \tilde{f}_{2}^{k_{2}+k_{3}} b_{2} \otimes \tilde{f}_{1}^{k_{1}+k_{2}-k_{3}} b_{1} & \left(k_{1} \geqq k_{3}\right) .\end{cases}
\end{aligned}
$$

Therefore we have

$$
\begin{aligned}
f^{\left(k_{1}, k_{2}, k_{j}\right)} & \equiv \tilde{f}_{1}^{k_{1}} \tilde{f}_{2}^{k_{2}+k_{3}} \tilde{f}_{1}^{k_{2}} u_{\infty} \\
& \longmapsto\left(u_{\infty} \otimes \tilde{f}_{1}^{k_{2}} b_{1} \otimes \tilde{f}_{2}^{k_{2}{ }^{+k_{3}}} b_{2} \otimes \tilde{f}_{1}^{k_{1}} b_{1}\right)^{*} .
\end{aligned}
$$

\section{Example 4.2.2.}

$$
\begin{array}{ll}
g=B_{2}, & I=\{1,2\}, \quad a_{\imath \jmath}=-2, \quad a_{\jmath \imath}=-1, \\
\beta_{1}=\alpha_{1}, \quad \beta_{2}=2 \alpha_{1}+\alpha_{2}, \quad \beta_{3}=\alpha_{1}+\alpha_{2}, \quad \beta_{4}=\alpha_{2} .
\end{array}
$$

We can calculate similarly, 


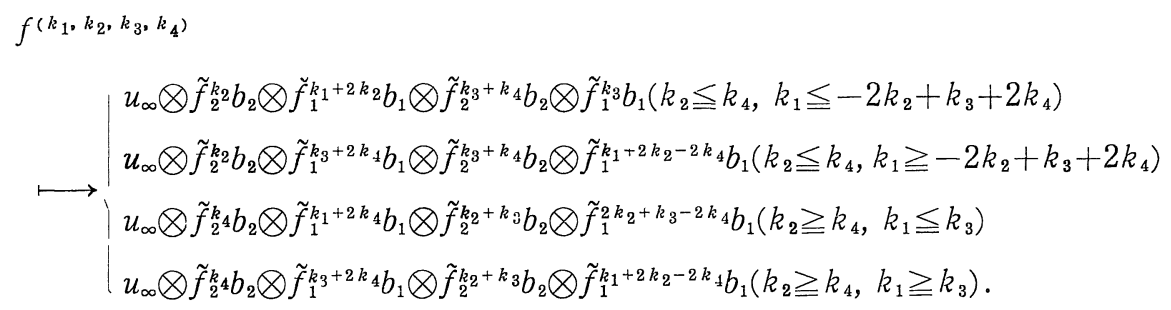

\section{References}

[1] Kashiwara, M., Crystallizing the $q$-analogue of universal enveloping algebras, Duke Math. J., 63 (1991), 465-516.

[2] - Grobal crystal base of quantum groups, RIMS preprint 756 (1991), to appear in Duke Math. J.

[3] - Crystal base and Littelmann's refined Demazure character formula, RIMS preprint 880 (1992).

[4] G. Lusztig, Quantum groups at roots of 1. Geom. Dedicata, 33 (1990), 89-113.

[5] - Finite dimensional Hopf algebras arising from quantized universal enveloping algebras, J. Amer. Math. Soc., 3 (1990), 257-296.

[6] - Canonical bases arising from quantized enveloping algebras, J. Amer. Math. Soc., 3 (1990), 447-498.

[7] Canonical bases arising from quantized enveloping algebras II, Progr. Theor. Phys. Suppl., 102 (1990), 175-201. 